\title{
Evaluation of Patient Positioning during Digital Tomosynthesis and Reconstruction Algorithms for Ilizarov Frames: A Phantom Study
}

\author{
Yuki Abe ${ }^{1}$, Makoto Shimada ${ }^{2}$, Yoshihiro Takeda ${ }^{3}$, Taisuke Enoki ${ }^{4}$, Kumiko Omachi $^{5}$, Shuji Abe ${ }^{6}$
}

\begin{abstract}
Aim: Metallic components from circular external fixators, including the llizarov frame, cause artefacts on X-rays and obstruct clear visualisation of bone detail. We evaluated the ability of tomosynthesis to reduce interference on radiographs caused by metal artefacts and developed an optimal image acquisition method for such cases.

Materials and methods: An llizarov frame phantom was constructed using rods placed on the bone for the purpose to evaluate the benefits of tomosynthesis. Distances between the rod and bone and the angle between the rod and X-ray tube orbit were set at three different levels. Filtered backprojection images were reconstructed using two different features of the reconstruction function: THICKNESS-- (CONTRAST4) and THICKNESS++ (METAL4); the first is suitable for improving contrast and the second is suitable for metal artefacts. The peak signal-to-noise ratio (PSNR) was used during image evaluation to determine the influence of the metallic rod on bone structure visibility.

Results: The PSNR increased as the angle between the metal rod and the X-ray tube orbit and the distance between the metallic rod and bone increased. The PSNR was larger when using THICKNESS-- (CONTRAST4) than when using THICKNESS++ (METAL4).

Conclusion: The optimal reconstruction function and image acquisition determined using the metallic rod in this study suggest that quality equal to that without the metallic rod can be obtained.

Clinical significance: We describe an optimised method for image acquisition without unnecessary acquisition repetition and unreasonable posture changes when the bone cannot be adequately visualised.

Keywords: Digital tomosynthesis, llizarov, Metal artefacts, Metallic rod, Peak signal-to-noise ratio, X-ray.

Strategies in Trauma and Limb Reconstruction (2020): 10.5005/jp-journals-10080-1446
\end{abstract}

\section{INTRODUCTION}

Digital tomosynthesis (DT) combines the benefits of digital imaging with those of computed tomography to provide three-dimensional (3D) structural information. It that can be obtained easily in conjunction with radiography using lower radiation doses and at a lower cost. However, DT reconstruction may produce inconsistent reconstructed images limited by a low signal-to-noise ratio from the superposition of several low-exposure projection images. ${ }^{1}$

The Ilizarov method has been established as a powerful technique for the management of various bone diseases and conditions. The evolutionary development of the llizarov method for bone lengthening and its current role have improved the quality of life of millions of people worldwide. ${ }^{2}$ Control and guidance of the bone shape-forming process remain the basis of the llizarov method. Follow-up of patients treated by the llizarov method involve weekly or biweekly standard plain anteroposterior and lateral radiographs with acquisition of additional radiographs of the centre of the distraction site. ${ }^{3}$ Radiologists need to measure the length of the lengthened bone column and remaining bonetransport defect to provide an estimate of the remaining treatment days. More importantly, radiologists must evaluate the quality of bone at the corticotomy site, which is known as the regenerated bone. ${ }^{4}$ In some cases, the quality of bone is obscured by artefacts from obstructing metal components of the circular external fixator. rod

Bone structures can be visualised better using DT in a single session because it is less affected by metal artefacts and enables the
${ }^{1-3}$ Department of Radiological Technology, Graduate School of Health Sciences, Okayama University, Okayama, Japan

${ }^{4}$ Department of Educational Collaboration, Health and Safety Sciences, Osaka Kyoiku University, Kashiwara, Osaka, Japan

${ }^{5}$ Department of Radiology, Osaka General Medical Center, Osaka, Japan

${ }^{6}$ Department of Radiology, Osaka Women's and Children's Hospital, Izumi, Osaka, Japan

Corresponding Author: Yuki Abe, Department of Radiological Technology, Graduate School of Health Sciences, Okayama University, Okayama, Japan, Phone: +81-90-7966-3577, e-mail: he422702@s.okayama-u.ac.jp

How to cite this article: Abe Y, Shimada M, Takeda Y, et al. Evaluation of Patient Positioning during Digital Tomosynthesis and Reconstruction Algorithms for Ilizarov Frames: A Phantom Study. Strategies Trauma Limb Reconstr 2020;15(1):1-6.

Source of support: Nil

Conflict of interest: None

acquisition of images at any height parallel to the DT bed. Even so with DT, the visibility of bone structures is affected by shadows from the metallic rod. No previous studies have reported the optimal acquisition method for avoiding such shadow artefacts. Moreover, a quantitative evaluation of images including metal shadows projected at the imaging site has not yet been conducted, and there are no indicators currently to help determine the optimal imaging and reconstruction methods. Therefore, in the present study, we 
aimed to evaluate the use of DT for reducing the interference caused by metal artefacts on bone radiographs and to develop the optimal image acquisition method for such cases.

\section{Materials and Methods}

\section{Phantom Specifications}

To evaluate the reduction of metal artefacts, we prepared a prosthetic phantom containing an artificial bone. The phantom was obtained commercially and it contained a metallic rod (Ilizarov, Smith \& Nephew, London, UK) placed on the bone.

\section{DT System}

The DT system (Sonialvision Safire II; Shimadzu Co., Kyoto, Japan) comprises an X-ray tube filter with a $0.6-\mathrm{mm}$ focal spot and a $362.8-\times$ 362.8-mm digital flat-panel detector composed of amorphous selenium. The source-to-isocentre and isocentre-to-detector distances were 980 and $1100 \mathrm{~mm}$ respectively (anti-scatter grid, focussed type; grid ratio, 12:1). DT was performed linearly with a total acquisition time of 5.0 seconds (effective dose: $47 \mathrm{kVp}, 1.25 \mathrm{mAs}$ ).

The reference radiation dose was that used in clinical practice because the clinical task was to assess the Ilizarov method. To sample the projection images during a single tomographic pass (projections, 74 ; acquisition angle, $40^{\circ}$ ) and reconstruct tomograms of the desired height, we used a $1024 \times 1024$ matrix with 32 bits (single-precision floating number) per image (pixel size, $0.279 \mathrm{~mm}$; reconstruction interval, $1 \mathrm{~mm}$ ).

\section{Image Acquisition}

Images were evaluated using the distance, angle, and reconstruction function that are assumed by the llizarov method. Figures $1 \mathrm{~A}$ to $C$ show an example of the distance between the metallic rod and the bone, which was set at three different levels: 45, 65, and $85 \mathrm{~mm}$. Figures $1 \mathrm{D}$ and $\mathrm{F}$ show an example of the angle between the metallic rod and the X-ray scanning direction, which was also set at three different angles: 10,25 , and $40^{\circ}$. Images were acquired five times under these settings.

\section{Reconstruction Function}

The reconstruction function of the filtered back-projection (FBP) method of the DT system (Sonialvision Safire II; Shimadzu Co., Kyoto, Japan) can be broadly divided into THICKNESS-- and THICKNESS++. The main difference between these two features is that THICKNESS++ is restricted compared to THICKNESS-- with regard to the position information for the height direction.

THICKNESS-- is available as five types: THICKNESS--, THICKNESS-- (CONTRAST), THICKNESS-- (CONTRAST2), THICKNESS-(CONTRAST4), and THICKNESS-- (CONTRAST6). THICKNESS++ is also available as five types: THICKNESS ++ , THICKNESS ++ (METAL), THICKNESS++ (METAL2), THICKNESS++ (METAL4), and THICKNESS++ (METAL6). When the number of these features increases (for example, from CONTRAST2 to CONTRAST4), the undershooting artefact around the metallic rod, which is generated around highly absorbent materials such as metal, is improved and the emphasis of the edge is weakened. Therefore, we selected the reconstruction function that best suited our purpose.

THICKNESS-- (CONTRAST4) and THICKNESS-- (METAL4) are used in clinical work at our hospital; therefore, these two features were adopted. The thickness for THICKNESS-- (CONTRAST4) was $4.1 \mathrm{~mm}$, and that for THICKNESS++ (METAL4) was $12.1 \mathrm{~mm}$. Because we wanted to examine how the thickness of the fault affects the image, we compared -- with ++. Even if the values of -- and ++ change, the fault thickness does not change.

\section{Evaluation}

The simplest and most widely used full-reference quality metric is the mean squared error (MSE), which is computed by averaging the squared intensity differences of distorted and reference image pixels, along with the related quantity of the peak signal-to-noise ratio (PSNR). MSE and PSNR are appealing because they are simple to calculate, have clear physical meanings, and are mathematically convenient in the context of optimisation. ${ }^{5}$

To evaluate the effects and severity of metal artefacts in each image featured in the in-focus plane, we calculated the PSNR. Image data were evaluated using Image J 1.36 (National Institutes of Health, Bethesda, MD, USA). The PSNR and MSE were calculated by the following equations:

$$
\begin{aligned}
\mathrm{PSNR}[\mathrm{dB}] & =10 \cdot \log _{10} \frac{\mathrm{MAX}}{\mathrm{MSE}} \\
\mathrm{MSE} & =\frac{1}{N} \sum_{i=1}^{N}\left\|X(i)-X^{\prime}(i)\right\|^{2}
\end{aligned}
$$

where MAX is the dynamic range of the pixel values (in this case, 65,535 for 16 -bit grayscale images), $i$ is the pixel number assigned to each pixel position, $X(i)$ is the pixel value in the original image, and $X^{\prime}(i)$ is the pixel number in the deteriorated image.

In this study, the original image was that of the bone phantom only and the deteriorated image was that of the bone phantom with a metallic rod. The size of the region of interest (ROI) during the evaluation of the feature (metal artefact) and background was $10 \mathrm{~mm} \times 10 \mathrm{~mm}$ (100 pixels). Therefore, there were $100 i$, from 1 to 100 .

Figures $1 \mathrm{G}$ and $\mathrm{H}$ show the measurement point, its enlarged view, and its pixel value in the bone phantom only and in the bone phantom with a metallic rod, respectively. The PSNR value in Figure $1 \mathrm{H}$, calculated using Figure $1 \mathrm{G}$, is 10.48 .

When the difference between the two images is large, the PSNR value decreases. Typically, a PSNR value of $\geq 40 \mathrm{db}$ makes it difficult to distinguish the deteriorated image from the original image, whereas a value of $\leq 20 \mathrm{db}$ makes the image unacceptable. ${ }^{6}$

The cross-section measurement was at the centre of the radius (85 $\mathrm{mm}$ from the top of the examination table). We set the point that split the radius into two (inside and outside, proximal, and distal). A point $30 \mathrm{~mm}$ away from the distal end of the radius was considered the centre of the ROI.

The effects of metal artefacts on each data set were assessed by a one-way analysis of variance and multiple comparisons test (i.e., the Tukey-Kramer test). The dependent variable was the PSNR; the independent variables were the reconstruction function, distance, and angle. A total of 15 samples without a metallic rod and 45 samples with a metallic rod were evaluated. Statistical analyses were performed using SPSS for Windows (version 22.0; IBM Corp., Armonk, NY, USA). $p<0.05$ was considered statistically significant.

The measurement value depended on the size of the ROI. A larger ROI indicated a larger signal change in the ROI. A smaller $\mathrm{ROI}$ indicated a larger measurement error in terms of statistical variation. The method of SNR determination using clinical images $(\text { AKIO OGURA })^{7}$ indicated that the position of the ROI was selected for uniformity of the signal intensity area and that the size of the $\mathrm{ROI}$ was more than $7 \times 7$ pixels. In this study, the diameter of the metallic rod was $10.0 \mathrm{~mm}$ based on the llizarov device. The objective 

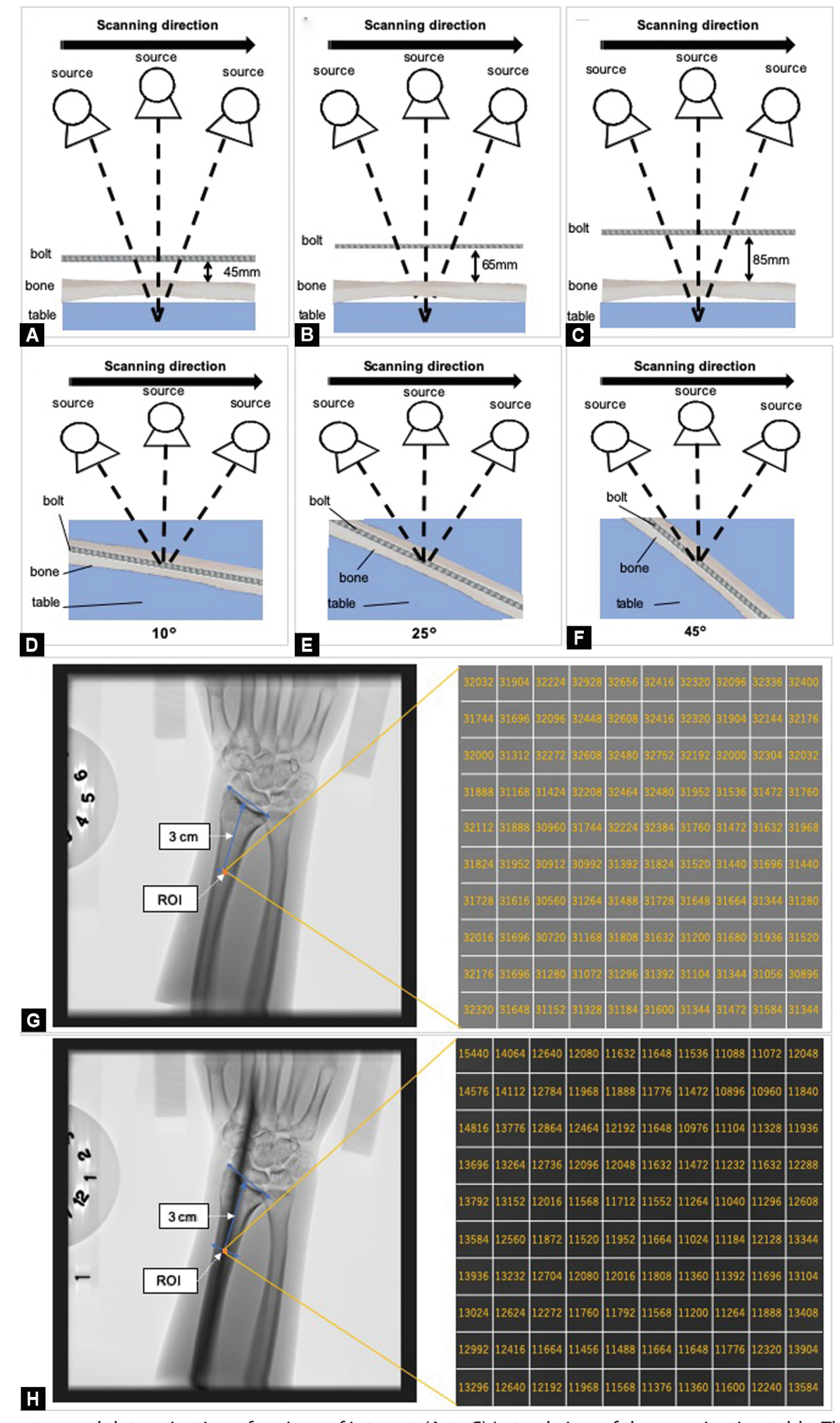

Figs $1 \mathrm{~A}$ to $\mathrm{H}$ : Phantom setup and determination of regions of interest. (A to C) Lateral view of the examination table. The distance between the metallic rod and bone was set at $45 \mathrm{~mm}$ (A), $65 \mathrm{~mm}$ (B), and $85 \mathrm{~mm}$ (C). (D to F) Vertical view of the examination table. The angle between the metallic rod and X-ray scanning direction was set at $10^{\circ}(\mathrm{D}), 25^{\circ}(\mathrm{E})$, and $40^{\circ}(\mathrm{F})$. The measurement point, its enlarged view, and corresponding pixel value in the case of the bone phantom only $(\mathrm{G})$. The bone phantom with a metallic rod and the PSNR value (calculated using $\mathrm{G})$ is $10.48(\mathrm{H})$. ROI, region of interest 
was to analyse the point with the highest influence on the shadow of the metallic rod. In preliminary experiments, when the ROI size became larger than $10.0 \mathrm{~mm}$, the difference between the PSNR of bone with and without the metallic rod became smaller. However, this was not the same for a uniform phantom. Therefore, it was necessary to minimise the influence of the positional shift of the measurement point. As a result, $10 \times 10$ pixel $(10.0 \mathrm{~mm} \times 10.0 \mathrm{~mm})$ ROIs were adopted.

\section{Results}

As described in the Methods section, we calculated the PSNR values by scanning at specific angles between the $X$-ray scanning direction and subjects (set at 10,25 , and $40^{\circ}$ ) using two different phantoms: a bone phantom and a bone phantom with a metallic rod. The results are shown in Table 1.

\section{Reconstruction Function}

The PSNR value of the reconstruction function THICKNESS-(CONTRAST4) was always higher than that of the function THICKNESS++ (METAL4). Even with the same angle and distance, using THICKNESS-- (CONTRAST4) led to more measurement points

Table 1: Average peak signal-to-noise ratio values obtained using digital tomosynthesis reconstruction algorithms

\begin{tabular}{ccc}
\hline $\begin{array}{l}\text { Distance/ } \\
\text { reconstruction function }\end{array}$ & $\begin{array}{c}\text { THICKNESS-- } \\
\text { (CONTRAST4) }\end{array}$ & $\begin{array}{l}\text { THICKNESS++ } \\
\text { (METAL4) }\end{array}$ \\
\hline $10^{\circ}$ angle between the metallic rod and X-ray scanning direction \\
Only phantom & 27.90 & 32.02 \\
$45 \mathrm{~mm}$ & 12.22 & 8.73 \\
$65 \mathrm{~mm}$ & 17.03 & 12.07 \\
$85 \mathrm{~mm}$ & 19.64 & 15.12 \\
$25^{\circ}$ angle between the metallic rod and X-ray scanning direction \\
Only phantom & 25.09 & 29.24 \\
$45 \mathrm{~mm}$ & 16.63 & 13.06 \\
$65 \mathrm{~mm}$ & 19.01 & 16.35 \\
$85 \mathrm{~mm}$ & 23.24 & 22.90 \\
$40^{\circ}$ angle between the metallic rod and X-ray scanning direction \\
Only phantom & 26.73 & 32.79 \\
$45 \mathrm{~mm}$ & 19.59 & 17.63 \\
$65 \mathrm{~mm}$ & 23.10 & 22.45 \\
$85 \mathrm{~mm}$ & 26.39 & 24.14 \\
\hline
\end{tabular}

in which a significant main effect was observed. Table 2 shows the measurement points with no significant difference at an angle and distance of $40^{\circ}$ and $85 \mathrm{~mm}$, respectively ( $40^{\circ}$ was the largest angle; $85 \mathrm{~mm}$ was the longest distance). Examples of images obtained with THICKNESS-- (CONTRAST4) and THICKNESS++ (METAL4) are shown in Figures $2 A$ and $B$, respectively.

\section{Distance}

We found that the PSNR increased as the distance between the metallic rod and the bone increased. The number of measurement
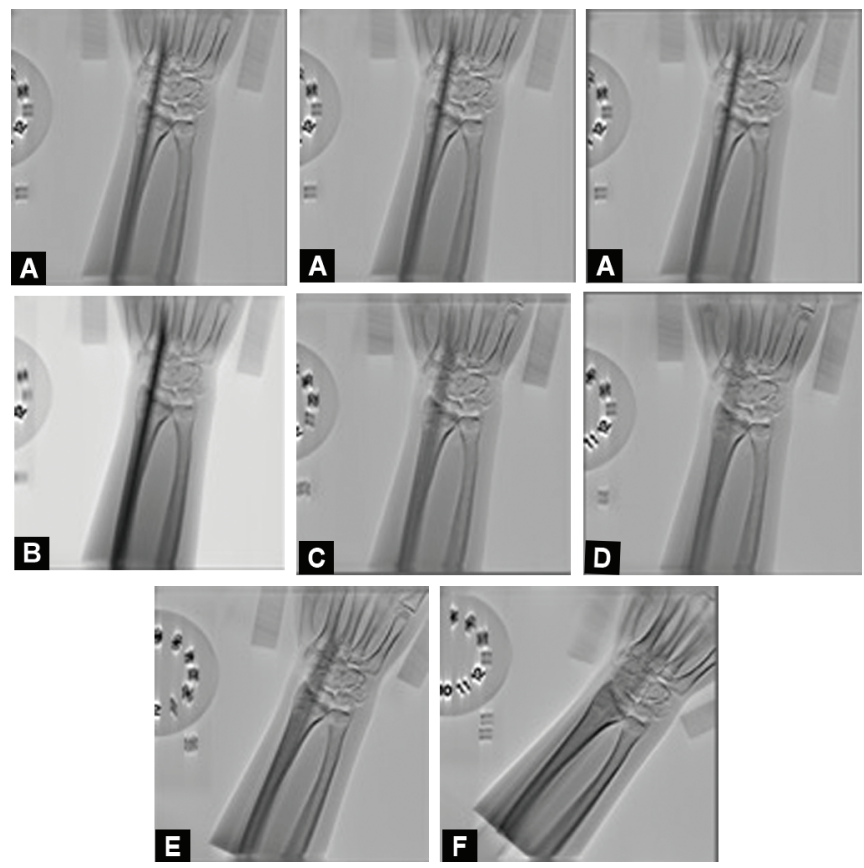

Figs $2 \mathrm{~A}$ to F: Examples of digital tomosynthesis images: (A) Images acquired at a $10^{\circ}$ angle and $45-\mathrm{mm}$ distance using THICKNESS-(CONTRAST4). The peak signal-to-noise ratio (PSNR) was 12.55; (B) Image acquired at a $10^{\circ}$ angle and $45-\mathrm{mm}$ distance using THICKNESS++ (METAL4). The PSNR was 10.48; (C) Image acquired at a $10^{\circ}$ angle and 65-mm distance using THICKNESS-- (CONTRAST4). The PSNR was 17.11 ; (D) Image acquired at a $10^{\circ}$ angle and $85-\mathrm{mm}$ distance using THICKNESS-- (CONTRAST4). The PSNR was 19.82; (E) Image acquired at a $25^{\circ}$ angle and $45-\mathrm{mm}$ distance using THICKNESS-- (CONTRAST4). The PSNR was 17.01; (F) Image acquired at a $40^{\circ}$ angle and $45-\mathrm{mm}$ distance using THICKNESS-- (CONTRAST4). The PSNR was 20.07

Table 2: Results of the multiple comparison analysis (Tukey-Kramer method)

\begin{tabular}{|c|c|c|c|c|}
\hline & $\begin{array}{l}40^{\circ}, 85 \mathrm{~mm}, \\
\text { THICKNESS-- } \\
\text { (CONTRAST4) }\end{array}$ & $\begin{array}{l}40^{\circ}, 85 \mathrm{~mm}, \\
\text { THICKNESS++ } \\
\text { (METAL4) }\end{array}$ & $\begin{array}{l}40^{\circ}, 65 \mathrm{~mm}, \\
\text { THICKNESS-- } \\
\text { (CONTRAST4) }\end{array}$ & $\begin{array}{l}25^{\circ}, 85 \mathrm{~mm}, \\
\text { THICKNESS-- } \\
\text { (CONTRAST4) }\end{array}$ \\
\hline $10^{\circ}, 85 \mathrm{~mm}$, THICKNESS-- (CONTRAST4) & a & $\mathrm{a}$ & n.s. & n.s. \\
\hline $25^{\circ}, 65 \mathrm{~mm}$, THICKNESS-- (CONTRAST4) & a & a & n.s. & n.s. \\
\hline $25^{\circ}, 85 \mathrm{~mm}$, THICKNESS-- (CONTRAST4) & n.s. & n.s. & n.s. & - \\
\hline $40^{\circ}, 45 \mathrm{~mm}$, THICKNESS-- (CONTRAST4) & a & a & n.s. & n.s. \\
\hline $40^{\circ}, 65 \mathrm{~mm}$, THICKNESS-- (CONTRAST4) & n.s. & n.s. & - & n.s. \\
\hline $40^{\circ}, 85 \mathrm{~mm}$, THICKNESS-- (CONTRAST4) & n.s. & - & n.s. & n.s. \\
\hline $25^{\circ}, 85 \mathrm{~mm}$, THICKNESS++ (METAL4) & a & n.s. & n.s. & n.s. \\
\hline $40^{\circ}, 65 \mathrm{~mm}$, THICKNESS++ (METAL4) & n.s. & n.s. & n.s. & n.s. \\
\hline $40^{\circ}, 85 \mathrm{~mm}$, THICKNESS ++ (METAL4) & n.s. & - & n.s. & n.s. \\
\hline
\end{tabular}

n.s.: no significant difference; ${ }^{a} p<0.05$ 
points showing a significant main effect also increased as the distance between the metallic rod and the bone increased. Table 2 shows the measurement points demonstrating that there was no significant difference in the combination of an $85-\mathrm{mm}$ distance and THICKNESS-- (CONTRAST4) (85 $\mathrm{mm}$ was the longest distance). Examples of images obtained with THICKNESS-- (CONTRAST4), a $10^{\circ}$ angle, and changes in the distance are shown in Figures $2 \mathrm{~A}, \mathrm{C}$, and D.

\section{Angle}

We found that the PSNR increased as the angle between the metallic rod and the X-ray scanning direction increased. The number of measurement points showing a significant main effect also increased as the angle between the metallic rod and the X-ray scanning direction increased. Table 2 shows the measurement points demonstrating no significant difference in the combination of a $40^{\circ}$ angle and THICKNESS-- (CONTRAST4) $\left(40^{\circ}\right.$ was the largest angle). Examples of images obtained with THICKNESS-(CONTRAST4), a 45- $\mathrm{mm}$ distance, and changes in the angle are shown in Figures $2 \mathrm{~A}, \mathrm{E}$ and $\mathrm{F}$.

\section{Images of the Bone Phantom Alone and Under Optimal Conditions}

The highest PSNR value of the bone phantom with the metallic rod was 26.39 with the combination of a $40^{\circ}$ angle, $85-\mathrm{mm}$ distance, and THICKNESS-- (CONTRAST4). Without the metallic rod, the PSNR value was 26.73 with the combination of a $40^{\circ}$ angle, no metallic rod, and THICKNESS-- (CONTRAST4). The Tukey-Kramer test indicated no significant difference between the two combinations ( $p \geq 0.05$ ).

\section{Discussion}

Our study showed that the reconstruction function, distance, and angle parameters are critical for obtaining the optimal imaging results for cases involving metallic rods that cannot be removed from the scanning field.

\section{Optimal Reconstruction Function}

When using the FBP method, the frequency limit is set in the depth direction ( $Z$ axis). In our study, THICKNESS++ (METAL4) produced a more restricted frequency range than THICKNESS-(CONTRAST4), indicating that it is restricted with regard to the positional information of the height direction. The use of a thinner fault thickness with THICKNESS-- (CONTRAST4) $(4.1 \mathrm{~mm})$ than with THICKNESS++ (METAL4) $(12.1 \mathrm{~mm})$ reduced the size of the high-absorption component (the metallic rod part). Therefore, the PSNR increased by changing the reconstruction function from THICKNESS++ (METAL4) to THICKNESS-- (CONTRAST4) using the same angle and distance. For cases in which metallic rods cannot be removed from the scanning field, the bone can be clearly seen by using the reconstruction function THICKNESS-- (CONTRAST4). In contrast, because THICKNESS++ (METAL4) provides a much more restricted frequency range than THICKNESS-- (CONTRAST4), it improves the undershooting artefacts around the metallic rod.

The reduction of artefacts is a distinctive advantage of our method. When using DT, artefacts occur as very low signals along the scanning direction around the edges of highly attenuating materials, such as metal prostheses or osteosynthetic materials. These artefacts are predominantly caused by a mismatch between the assumptions of the reconstruction algorithm (ideal monochromatic beam) and reality (wide spectral range). The limited scanning angle (in this study, $40^{\circ}$ ) also contributes to this effect, but to a much less extent. When observing the area around the metallic rod, a clearer image was obtained using THICKNESS++ (METAL 4) rather than THICKNESS-- (CONTRAST4). With DT, images from various reconstruction functions can be obtained from the image data without the need to repeat the acquisition. Therefore, when rods cannot be placed outside the scanning field, the images should be reconstructed using THICKNESS-- (CONTRAST4). However, when the observation is performed near to the metallic rod, the images should be reconstructed using THICKNESS++ (METAL4).

\section{Optimal Image Acquisition}

The degradation of CT images occurs due to the interaction between the polyenergetic X-ray beam and dense structures, thus creating two distinctive effects. The first effect is generalised noise proportional to the overall amount of metal present on the axial slice. It is seen as a fine, dark, bright streak on the image. In this case, the artefact is distributed fairly and proportionately across the image. The second effect, which is due to pairing between the rods and struts, causes a more noticeable broad dark streak with surrounding bright edges and is a function of the helical manner in which the scan is acquired.

Figure $3 \mathrm{~A}$ shows the relationship among the X-ray tube, external fixture, and X-ray detector for helical CT, and an example of metallic rod projection on a helical CT image. During the CT scanning process, the source completes a full rotation and will include within a scan of the metallic rod. ${ }^{8}$ Therefore, the bone cannot be adequately observed because of the shadows created by the metallic rod.

DT image quality depends on numerous factors, such as the size, shape, density, atomic number, and position of the metal objects, as well as the size and shape of the object's cross-section. Particularly for implants manufactured from metals, the effects of beam hardening and scatter are high; hence, noise-induced streaking artefacts affect image quality. During image acquisition with DT, the acquisition angle is $40^{\circ}$. If rods cannot be removed from the scanning field, then the DT scan enables one to observe the bone without metal artefacts (Fig. 3B). Nevertheless, even with DT, the visibility of bone structures is affected by artefacts from the metallic rod, thereby adversely affecting image quality. In these cases, dispersing the influence of the metallic rod appears to be a

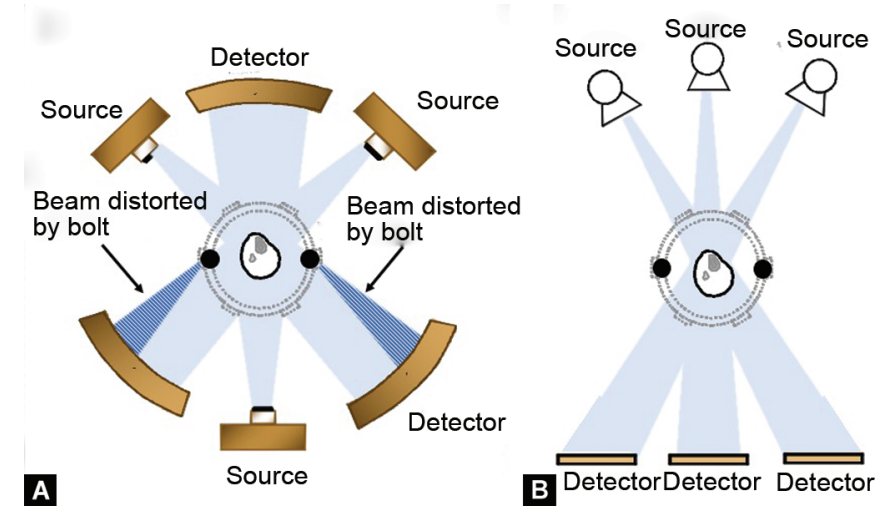

Figs $3 \mathrm{~A}$ and $\mathrm{B}$ : (A) Helical nature of the computed tomography scanning process; (B) Digital tomosynthesis scanning process 
promising approach to reducing artefacts stemming from metals with a relatively high atomic number.

In our study, when the distance between the metallic rod and bone increased, the metal rod artefact was dispersed in the vertical direction (X-ray scanning direction) as the angle between the metallic rod and X-ray scanning direction increased. As a result, the metal artefact was reduced and the PSNR increased. Therefore, the metal shadows dispersed and the PSNR increased when the distance between the metallic rod and bone increased, and when the angle between the metallic rod and X-ray tube orbit increased. The angle between the metallic rod and X-ray scanning direction should be larger but this is usually limited in actual clinical practice. In the case of the llizarov fixator used for the lower leg, considering the bed width in the present study, an angle of $40^{\circ}$ was the limit.

\section{Conclusion}

In bone phantoms with metallic rods, the PSNR was highest (26.39) with the following conditions: THICKNESS-- (CONTRAST4), $85-\mathrm{mm}$ distance, and $40^{\circ}$ angle. This compares very favourably to results without a metallic rod when using the function THICKNESS-(CONTRAST4); here the PSNR was 26.73.

The Tukey-Kramer test showed no significant differences between the following conditions: [THICKNESS-- (CONTRAST4)/ no metallic rod $/ 40^{\circ}$ angle] and [THICKNESS-- (CONTRAST4) $/ 85-\mathrm{mm}$ distance $/ 40^{\circ}$ angle] $(p<0.05)$. Therefore, to accurately describe findings or to identify potential obstacles that may arise during treatment, one should try to maintain the same image quality as without the metallic rod. For external fixation with the llizarov method, radiologists do not need to perform repeated imaging because one-time imaging with DT may provide an optimal image. Because DT image quality is based on the angle, using angles such as pronation or supination in the reference line or the angle of the measurement site with respect to the DT bed can lower the risk of interference. This suggests that images with high reproducibility can always be obtained as an output.

One limitation of our study was the image quality assessment. Sometimes, the PSNR is not very well-matched to the perceived visual quality. ${ }^{9-12}$ Although efforts are being made to produce an objective scoring system to assess the quality of digital images in general, to our knowledge no such score exists for radiography currently. Therefore, we performed an evaluation using the PSNR, which is, at present, considered an optimal full-reference quality metric.

\section{Clinical Significance}

As our method was based on the measurement of the distance between the metallic rod and bone using a plain radiograph, this helped to set the angle between the metallic rod and X-ray scanning direction which, in turn, enabled us to obtain an image equivalent to the one without the metallic rod. The use of this method can provide optimal images without positional changes or unreasonable posture changes, from a single acquisition session (without unnecessary repetition of image acquisition). This can benefit patients greatly.

\section{References}

1. Gomi T, Sakai R, Goto M, et al. Evaluation of digital tomosynthesis reconstruction algorithms used to reduce metal artefacts for arthroplasty: a phantom study. Phys Med 2017;42:28-38. DOI: 10.1016/j.ejmp.2017.07.023.

2. Gubin AV, Borzunov DY, Malkova TA. The llizarov paradigm: thirty years with the llizarov method, current concerns and future research. Int Orthop 2013;37(8):1533-1539. DOI: 10.1007/s00264-013-1935-0.

3. Young JW, Kovelman H, Resnik CS, et al. Radiologic assessment of bones after llizarov procedures. Radiology 1990;177(1):89-93. DOI: 10.1148/radiology.177.1.2399344.

4. Tresley J, Schoenleber SJ, Singer AD, et al. "Ilizarov" external fixation: what the radiologist needs to know. Skeletal Radiol 2015;44(2): 179-195. DOI: 10.1007/s00256-014-2001-8.

5. Wang Z, Bovik AC, Sheikh HR, et al. Image quality assessment: from error visibility to structural similarity. IEEE Trans Image Process 2004;13(4):600-612. DOI: 10.1109/tip.2003.819861.

6. Yahata K, Hada T, Kunitake Y, et al. Evaluation of JPEG and JPEG2000 compression endoscope medical image. Japan J Med Informat 2004;24(2):291-296.

7. Ogura A, Miyati T, Kobayashi M, et al. Method of SNR determination using clinical images. Nihon Hoshasen Gijutsu Gakkai Zasshi 2007;63(9):1099-1104.

8. Peña-Solórzano CA, Dimmock MR, Albrecht DW, et al. Effect of external fixation rod coupling in computed tomography. Strategies Trauma Limb Reconstruction 2018;13(3):137-149. DOI: 10.1007/s11751018-0318-x.

9. Teo PC, Heeger DJ. Perceptual image distortion. Proc SPIE 1994;2179:127-141.

10. Eskicioglu AM, Fisher PS. Image quality measures and their performance. IEEE Trans Commun 1995;43(12):2959-2965. DOI: 10.1109/26.477498.

11. Eckert MP, Bradley AP. Perceptual quality metrics applied to still image compression. Signal Processing 1998;70(3):177-200. DOI: 10.1016/ S0165-1684(98)00124-8.

12. Gao F, Tao D. Learning to rank for blind image quality assessment. IEEE Trans Neural Netw Learn Syst 2015;26(10):2275-2290. DOI: 10.1109/ TNNLS.2014.2377181. 